\title{
Optical correlation by use of partial phase-only modulation with VGA liquid-crystal displays
}

\author{
Ignasi Labastida, Arturo Carnicer, Estela Martín-Badosa, Santiago Vallmitjana, and \\ Ignacio Juvells
}

\begin{abstract}
The development of liquid-crystal panels for use in commercial equipment has been aimed at improving the pixel resolution and the display efficiency. These improvements have led to a reduction in the thickness of such devices, among other outcomes, that involves a loss in phase modulation. We propose a modification of the classical phase-only filter to permit displays in VGA liquid-crystal panels with a constant amplitude modulation and less than a $2 \pi$ phase modulation. The method was tested experimentally in an optical setup. (C) 2000 Optical Society of America

OCIS codes: $200.4740,100.4550,120.5060,230.3720$.
\end{abstract}

\section{Introduction}

Liquid-crystal displays (LCD's) that have been removed from commercial video projectors are commonly used as spatial light modulators in hybrid optoelectronic setups for optical correlation. ${ }^{1,2}$ The development of these liquid-crystal panels has focused on increasing their pixel resolution through a reduction in the panels' thickness and in their pixel pitch. For optical setup design purposes the pixelpitch decrease is an advantage because it involves a reduction in the total length of the setup. However, some such devices are not thick enough to achieve a phase modulation of $2 \pi \mathrm{rad}$.

To display a phase-only filter (POF) on LCD's, we demonstrate that the same correlation results can be obtained if the absolute value of the phase is taken. For that purpose, we require an operating curve with a constant amplitude modulation and at least a $\pi$-rad phase modulation when the maximum number of gray levels available is used. Other authors have tried to overcome the limited phase modulation of the LCD by means of different approaches, e.g., changing the operating wavelength of the setup ${ }^{3}$ or using a double-pass configuration. ${ }^{4}$

The authors are with the Departament de Física Aplicada i Optica, Universitat de Barcelona, Avinguda Diagonal 647, E08028 Barcelona, Spain. I. Labastida's e-mail address is nasi@optica. ub.es.

Received 10 May 1999; revised manuscript received 2 August 1999.

0003-6935/00/050766-04\$15.00/0

(C) 2000 Optical Society of America
We used two LCD's removed from a commercial VGA video projector. With these devices, we did not obtain the desired configuration with linearly polarized light. But, as was pointed out in Refs. 5-7, we achieved a suitable operating curve with elliptically polarized light. We built a converging VanderLugt correlator with two LCD's to validate the method. Optical correlations were obtained with the modified POF displayed on a LCD in an elliptically polarized light configuration.

\section{Liquid-Crystal Display Characterization}

The characterization of the liquid-crystal panels is focused mainly on the way in which these devices modulate light, depending on the gray level assigned to each pixel. Generally, amplitude and phase modulation are coupled in the sense that both the amplitude and the phase of light passing through spatial light modulators are modified. However, it is possible to find some operational conditions under which one characteristic remains mainly constant, whereas the other one changes considerably. These are called the amplitude-mostly configuration and the phase-mostly (PM) configuration, ${ }^{6,8}$ respectively. There is also a third configuration that often is used because of its high values of contrast: the highcontrast (HC) configuration. ${ }^{6}$ Although it introduces an important phase modulation, the contrast ratio is much higher than that obtained by use of an amplitude-mostly mode. On the other hand, using a PM configuration with a high constant transmittance maximizes the light efficiency of the optical arrangement because light absorption is minimal.

When placing a LCD between two polarizers dif- 


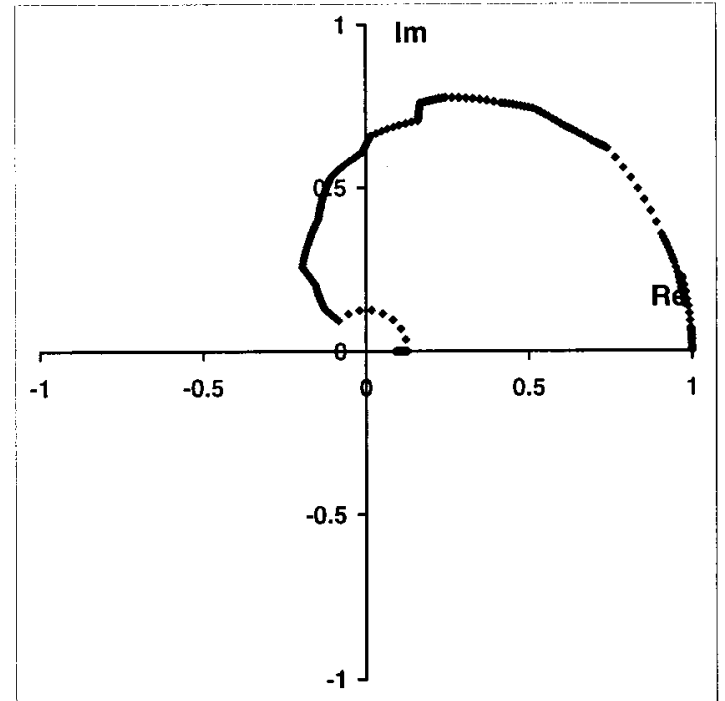

Fig. 1. HC operating curve. Re, real; Im, imaginary.

ferent configurations can be obtained, depending on the voltage applied and the orientation angles of the polarizers. The voltage applied depends on the gray level assigned to each pixel and on the position of the potentiometer controls, which regulate brightness, contrast, and color, of the video projector. These controls have to be set in a compromise position when a single video projector addresses two LCD's, and the curve search has to be carried out by rotation of the polarizers. This limitation reduces the acquisition of different operating curves.

In our study two LCD's that were removed from a commercially available VGA Epson video projector (Model EMP-3000) were characterized to find a HC configuration for the input device and a PM one for the filter. The characterization consists of determining how the LCD modulates the amplitude and the phase of the light. The amplitude modulation is the square root of the ratio between the intensities before and after the light passes through the panel. The phase modulation is obtained with an interferometric procedure that is based on the measurement of the relative phase shift between several pairs of gray values displayed on the LCD when it is placed in one arm of a Mach-Zehnder interferometer.5,9

We show two interesting resultant configurations: The first (Fig. 1) corresponds to a HC mode in which the device transmittance (amplitude squared) increases with the gray level and reaches a normalized maximum value of 1 for a gray level of 255 . The contrast of the modulator is approximately 125:1, which is high enough to display the input image. The second (Fig. 2) exhibits the best PM operating curve. It is not advisable to use configuration 2 to display a POF for several reasons: (i) The phase modulation reaches only $1.6 \pi$ rad. (ii) The amplitude modulation is not constant (the minimum value of the amplitude modulation is approximately $43 \%$ of the maximum amplitude obtained). (iii) If we use

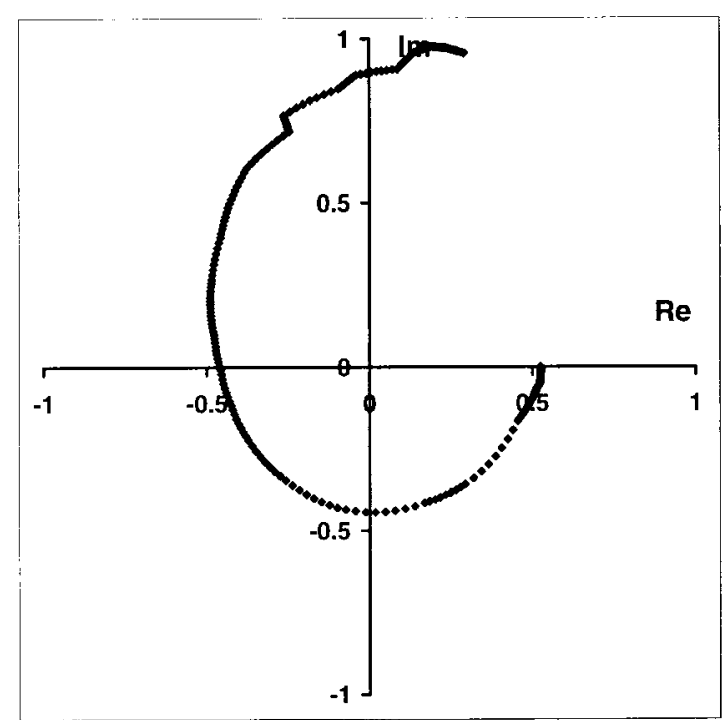

Fig. 2. Phase-mostly operating curve.

only the $[0-\pi]$ range, the transmittance is too low to yield good correlation results, and we lose bandwidth (104 gray levels).

Both curves (Figs. 1 and 2) were obtained by use of linearly polarized light at the input and the output of the LCD's. Nevertheless, if we use elliptically polarized light instead it is possible to achieve a configuration with just phase modulation. ${ }^{5}$

To obtain a suitable phase-only configuration with elliptically polarized light, we have to substitute the two linear polarizers that are at the input and the output sides of the LCD by two pairs of linear polarizers plus a $\lambda / 4$ plate. The fast axes of these plates must be oriented parallel to the corresponding molecular directors at each side of the panel. The input polarizer has to be rotated by an angle $\theta$ from the fast axis of the input plate, whereas the output polarizer must be oriented at an angle $-\theta$ relative to the fast axis of the output plate. ${ }^{7}$

For such conditions, we found an angle of $\theta=35^{\circ}$ for which the operating curve (Fig. 3) makes use of the 256 gray levels available, producing phase modulation and a constant transmittance. Although with this operational mode the phase modulation reaches only $\pi$ rad, we show in Section 3 a way to overcome this drawback for correlation purposes.

\section{Implementation of Phase-Only Filters with $[0-\pi]$ Modulation}

We observed that our devices can operate in a phaseonly modulation configuration in the range $[0-\pi]$ with constant amplitude and by use of the entire dynamic range of the image. For such conditions, we show that it is possible to achieve the same correlations as were obtained with an ordinary POF. ${ }^{10}$ However, with this $[0-\pi]$ curve it would be possible to implement binary masks by use of the points that correspond to the 0 and the $\pi$ modulations. Let $h(x, y)$ be the reference to detect, and let $H(u, v)=$ 


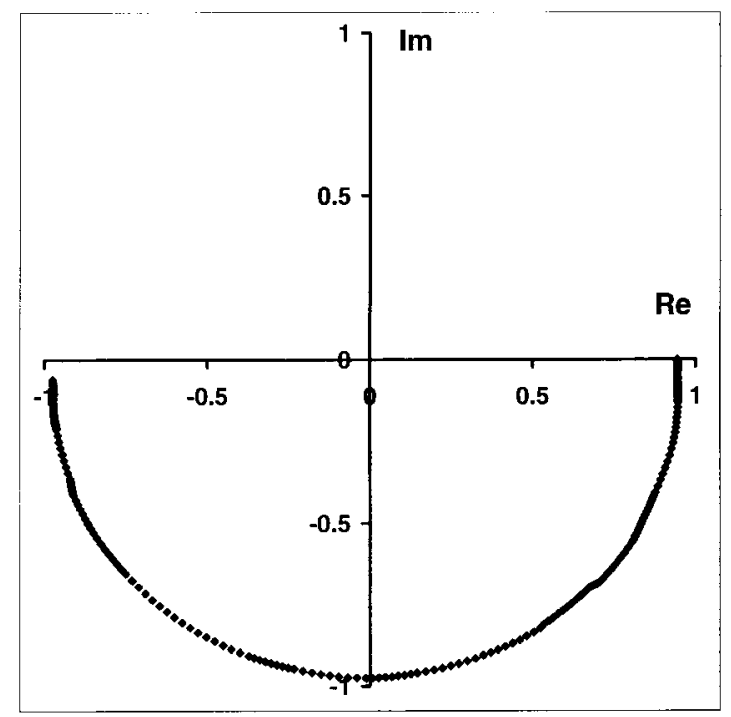

Fig. 3. LCD $[0-\pi]$ phase-only modulation operating curve.

$|H(u, v)| \exp [-i \phi(u, v)]$ be its Fourier transform. The POF is defined as $H_{\mathrm{POF}}(u, v)=\exp [-i \phi(u, v)]$. We then introduce the $\pi$ phase-only filter ( $\pi$-POF) as

$$
\begin{aligned}
H_{\pi-\mathrm{POF}}(u, v) & =\exp [-i|\phi(u, v)|] \\
& =\cos [\phi(u, v)]-i|\sin [\phi(u, v)]|,
\end{aligned}
$$

The function $f(x)=|\sin (x)|$ can be written as a Fourier series, as follows:

$$
\begin{aligned}
|\sin (x)|= & \frac{2}{\pi}-\frac{4}{\pi}\left[\frac{1}{3} \cos (2 x)+\frac{1}{15} \cos (4 x)\right. \\
& \left.+\frac{1}{35} \cos (6 x)+\ldots\right],
\end{aligned}
$$

and therefore the phase codified in the filter can be written as

$$
\begin{aligned}
\exp [-i|\phi(u, v)|]= & \cos [\phi(u, v)]-\frac{2 i}{\pi}+\frac{4 i}{3 \pi} \\
& \times \cos [2 \phi(u, v)]+\ldots
\end{aligned}
$$

To avoid the diffraction of all the terms superimposed on the zero order, we introduce a linear phase shift into the expression for the filter:

$$
\begin{aligned}
\exp [-i \mid \phi(u, v)+ & 2 \pi a u \mid] \\
=\cos [\phi(u, v)+2 \pi a u]-\frac{2 i}{\pi}+\frac{4 i}{3 \pi} & \\
& \times \cos [2 \phi(u, v)+2 \pi(2 a) u]+\ldots .
\end{aligned}
$$

By analyzing Eq. (4), we can obtain information about the filter reconstruction. Using the $\pi-\mathrm{POF}$, we have an on-axis constant term, and symmetrically arranged from it at a distance of $x= \pm a$ are the phaseonly reconstructions of the reference. These images have conjugated phases. There are also higherorder harmonics with decreasing energy at the points $x= \pm 2 a, \pm 4 a, \pm 6 a, \ldots$. Therefore it is expected that we will find the correlation and the convolution between the scene and the target situated symmetrically from the reconstruction of the scene.

\section{Optical Results}

To test our method experimentally, we built an optical setup that is based on the converging VanderLugt correlator, which is a modification of the classical $4 f$ architecture, ${ }^{11}$ because of its versatility in modifying the scale of the Fourier transform without a change in either the lenses or the distances between them. Two LCD's are used to display the images: one at the input plane and the other at the Fourier plane.
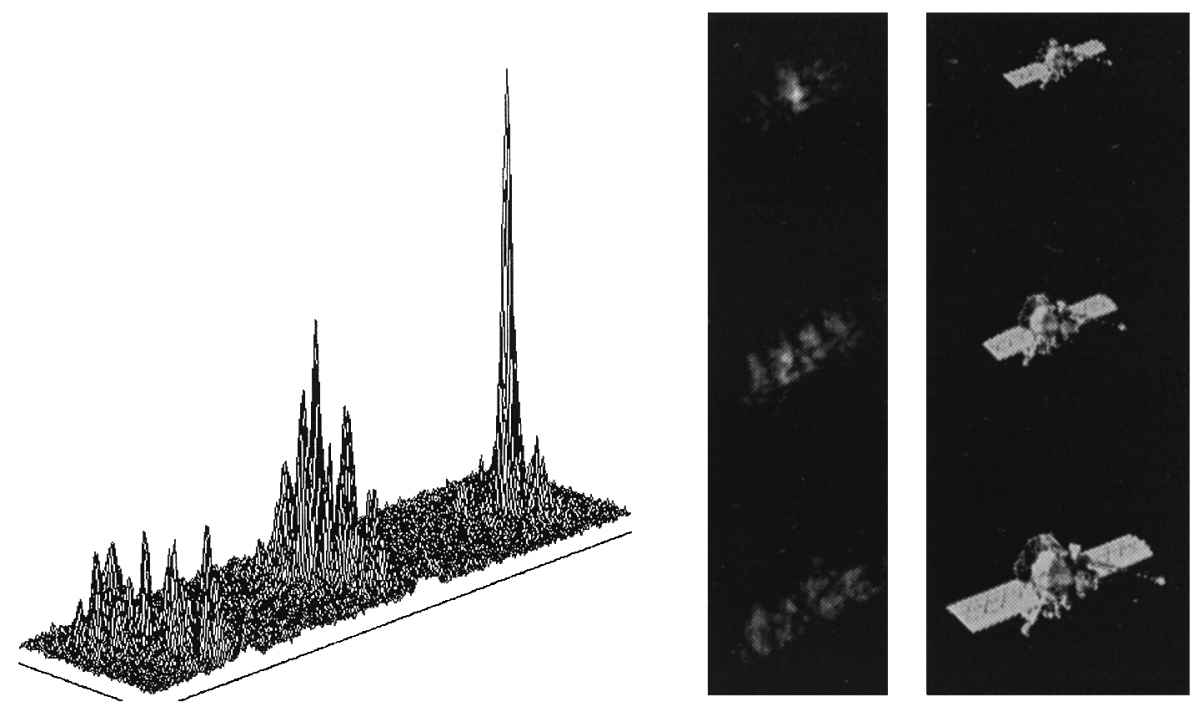

Fig. 4. Correlation between the scene and the smallest satellite. 
A VGA card stores a 24-bit red-green-blue image of $640 \times 480$ pixels. The red and the blue channels yield the information corresponding to the filter and the scene, respectively, whereas the green channel remains at zero. The VGA card is connected to the video projector, and the signals are sent to the red and the blue panels.

The input image used in the experiments comprises three satellites of different sizes. The original scene is $512 \times 512$ pixels and 8 bits/pixel ( 256 gray levels). By adding zero-valued points and losing some rows, we have an image displayed on the LCD that is $640 \times 480$ pixels to adapt its size to the VGA resolution. On the right-hand side of Fig. 4, we show the significant area of the scene. The input image is displayed on the first LCD in the HC configuration, whereas the filter is displayed on the second LCD with the $[0-\pi]$ phase-only modulation operating curve.

The $\pi$-POF of the target was computed with the phase introduced to the scene by the HC configuration of the first LCD taken into account. Figure 4 shows the optical correlation between the scene and the smallest satellite by use of the $\pi$-POF matched to it.

\section{Conclusions}

We have proposed a method for displaying a POF on a LCD with a less than $2 \pi$-rad phase modulation. By using elliptically polarized light, we have achieved a configuration with constant amplitude modulation and $\pi \mathrm{rad}$ in phase modulation. We have designed a filter to be displayed in that configuration to obtain the same correlation results that would be obtained with the classical POF. Experimental results have been obtained in a converging VanderLugt correlator that verify the method.
This study was supported by the CICYT (Comisión Interministerial de Ciencia y Tecnología) under project TAP97-0454.

\section{References}

1. D. A. Gregory, "Real-time pattern recognition using a modified liquid-crystal television in a coherent optical correlator," Appl. Opt. 25, 467-469 (1986).

2. A. Stoianov, C. Soutar, and A. Graham, "High-speed fingerprint verification using an optical correlator," in Optical Pattern Recognition IX, D. P. Casasent and T. Chao, eds., Proc. SPIE 3386, 242-252 (1998).

3. T. L. Kelly and J. Munch, "Wavelength dependence of twisted nematic liquid crystal phase modulators," Opt. Commun. 156, 252-258 (1998)

4. M. Yamauchi and T. Eiju, "Phase modulation capability of thin twisted nematic liquid crystal panels at double-pass configurations," Opt. Rev. 2, 24-27 (1995).

5. J. L. Pezzaniti and R. A. Chipman, "Phase-only modulation of a twisted nematic liquid-crystal TV by use of the eigenpolarization states," Opt. Lett. 18, 1567-1569 (1993).

6. C. Soutar and S. E. Monroe, Jr., "Selection of operating curves of twisted-nematic liquid-crystal televisions," in Advances in Optical Information Processing VI, D. R. Pape, ed., Proc. SPIE 2240, 280-291 (1994).

7. J. A. Davis, I. Moreno, and P. Tsai, "Polarization eigenstates for twisted-nematic liquid-crystal displays," Appl. Opt. 37, 937-945 (1998)

8. K. Lu and B. E. A. Saleh, "Theory and design of the liquid crystal TV as an optical spatial phase modulator," Opt. Eng. 29, 240-246 (1990).

9. E. Martín-Badosa, A. Carnicer, I. Juvells, and S. Vallmitjana, "Liquid crystal devices complex modulation characterization by interferometric data correlation," Meas. Sci. Technol. 8, 764-772 (1997).

10. J. L. Horner and P. D. Gianino, "Phase-only matched filtering," Appl. Opt. 23, 812-816 (1984).

11. D. Joyeux and S. Lowenthal, "Optical Fourier transform: what is the optimal setup?" Appl. Opt. 21, 4368-4372 (1982). 